\title{
An Implantable Synthetic SPECT Lesion: A Bridge from Phantom to Reality
}

\author{
M. Bret Abbott, Lars R. Furenlid, Don W. Wilson, Gail D. Stevenson, James M. Woolfenden, and Harrison H. Barrett \\ Center for Gamma-Ray Imaging, Department of Radiology, University of Arizona, Tucson, Arizona
}

\begin{abstract}
Small-animal imaging systems are often characterized using phantoms, which may not predict performance in clinical applications. An implantable synthetic SPECT lesion would facilitate characterization of lesion detectability in a living animal. Methods: Anion-exchange columns with bed volumes of 100$300 \mathrm{~nL}$ were constructed from medical-grade polyvinyl chloride tubing and resin. The columns were tested in an excised mouse femur and implanted in the femur of a living mouse. Imaging was performed using a prototype dual-modality SPECT/CT system. Results: Activity of 7.4-22.2 MBq (0.2-0.6 mCi) localized within the synthetic lesion. The synthetic lesions were reused multiple times. Mice tolerated the implanted columns without complications for up to 8 wk. Conclusion: A reusable, synthetic SPECT lesion was constructed and implanted in the femur of a living mouse. The synthetic lesion is useful for the development of imaging schemes and for more realistically evaluating imagingsystem performance in the context of a living animal.
\end{abstract}

Key Words: small-animal SPECT; phantom; synthetic lesion; implantable device

J Nucl Med 2007; 48:1796-1799

DOI: 10.2967/jnumed.107.046037

$\mathbf{T}$ he imaging of small-animal models of disease is an evolving science (1-5). Although dedicated small-animal imaging systems, including SPECT, PET, MRI, and CT, are commercially available, development of new small-animal systems with improved performance continues at a rapid pace. Small-animal imaging systems face additional constraints and limitations in spatial resolution and sensitivity relative to human imaging (6-8). Imaging phantoms are commonly used to assess equipment function and performance.

However, phantoms typically do not directly measure imaging-system performance of a clinical or biomedical task. Small-animal imaging systems pose additional technical problems, such as animal immobilization and anesthesia, image coregistration, and validation, which often cannot be adequately addressed with typical phantom studies.

\footnotetext{
Received Apr. 18, 2007; revision accepted Aug. 7, 2007.

For correspondence or reprints contact: M. Bret Abbott, MD, PhD, c/o Corrie Thies, Department of Radiology, P.O. Box 245067, Tucson, Arizona 85724-5067.

E-mail: mbamdphd@yahoo.com

COPYRIGHT @ 2007 by the Society of Nuclear Medicine, Inc.
}

An implantable synthetic SPECT lesion would bridge the gap between phantom studies and live small-animal imaging. An ideal synthetic SPECT lesion would have known size and activity ranges, be made of biocompatible materials, and be reusable. Incorporation of a synthetic lesion into a live animal would allow standardized imaging studies in the context of living tissues, with all of the real-life challenges of background activity, anesthesia, physiologic monitoring, and animal positioning. A rechargeable synthetic lesion would minimize the number of animals used during technique development. The known size, location, and activity range of a synthetic SPECT lesion could facilitate development and refinement of quantitative reconstruction algorithms, automated lesion detection, size-estimation tasks, and coregistration algorithms in multimodality imaging schemes.

\section{MATERIALS AND METHODS}

\section{Catheter Assembly}

Anion-exchange chromatography beads with quaternary amine groups were selected to provide fixed ionic binding sites for negatively charged ions in solution such as ${ }^{99} \mathrm{mcO}_{4}{ }^{-}$. Two to $6 \mathrm{AG}$ $1-\mathrm{X} 2,50$ - to 100-mesh anion-exchange chromatography beads (Bio-Rad) were placed inside a 5-mm length of polyvinyl chloride tubing (Scicom, Inc.) with a 0.023-in internal diameter and a 0.039-in external diameter. Medical-grade polyvinyl chloride tubing with a 0.01 -in internal diameter and a 0.030-in external diameter (Small Parts) was then inserted into each end of the slightly elastic tubing containing the chromatography beads. The joints between the inner and outer tubing were sealed with a suspension of polyvinyl chloride resin in $45 \%-60 \%$ methylethyl ketone, $25 \%-40 \%$ tetrahydrofuran, $5 \%-10 \%$ cyclohexanone, and 0\%-5\% acetone (Oatey Regular Clear Cement).

\section{Catheter Loading}

The anion-exchange beads were loaded with ${ }^{99} \mathrm{~m} \mathrm{TcO}_{4}{ }^{-}$by flushing the catheters with an aqueous solution of sodium pertechnetate followed by $0.5 \mathrm{~mL}$ bacteriostatic water. For external catheters, bound activity was measured in a well counter. For indwelling catheters, bound activity was estimated from the difference between the activity injected and the activity recovered after flushing of the catheter.

\section{Ex Vivo Catheter Placement}

An excised femur was obtained from a mouse sacrificed for other purposes. A hole was hand-drilled in the lateral distal metaphysis of the femur with an 18-gauge needle. A second 18-gauge 
hole was hand-drilled in the lateral mid-diaphysis. The intervening marrow space was cleared by passage of a curved needle. The catheter containing 5 beads was threaded through the marrow space until the bead chamber was located within the distal metaphysis.

\section{Catheter Implantation}

Experimental animals were handled and treated according to protocols approved by the Institutional Animal Care and Use Committee. Mice of approximately $25 \mathrm{~g}$ were gas-anesthetized (4\% isoflurane for induction; $1 \%-2 \%$ for maintenance), and the right leg and nape of the neck were shaved, sterilely prepared with alcohol and Betadine (povidone iodine solution; The Purdue Fredrick Co.), and draped. The skin over the lateral femur was incised with a scalpel. The lateral distal femur was exposed by blunt dissection between the vastus lateralis muscle bundles. Eighteen-gauge holes were hand-drilled in the distal metaphysis and mid-diaphysis, and a gas-sterilized catheter containing 4 beads was threaded into the marrow space. Bone wax was used to control blood loss. The skin at the nape of the neck was incised, and the catheter ends were tunneled through the subcutaneous tissues from the leg to the incision. The catheter was flushed, and the incisions were closed with methylmethacrylate glue. Buprenorphine $(0.06$ $\mathrm{mg}$ ) was injected subcutaneously perioperatively and the following morning for pain control. Recovery was facilitated by intradermal injection of warm normal saline and use of an external heat source, Nutri-Cal (dietary supplement; EVSCO), and water.

\section{Dual-Modality Imaging}

The dual-modality imager of the Center for Gamma-Ray Imaging incorporates a cadmium zinc telluride (CZT) detector module for planar and SPECT $\gamma$-ray imaging (9). The CZT detector is mated with a 7-mm-thick tungsten collimator with a $64 \times 64$ array of $260 \times 260 \mu \mathrm{m}$ square bores on a $380-\mu \mathrm{m}$ pitch aligned with the CZT pixel array. The collimator efficiency is $7 \times 10^{-5}$. Spatial resolution for both scintigraphy and SPECT is about $0.5 \mathrm{~mm}$. The $\mathrm{X}$-ray system consists of a microfocal X-ray source (Oxford Instruments) and a phosphor-coupled charged-coupled device camera (MedOptics). The CT and SPECT systems share a common rotary stage that allows geometric coregistration of CT and SPECT images (10).

Planar X-ray images were obtained as one-hundred eighty $1,024 \times$ 1,024 pixel projections over $360^{\circ}$ with $2^{\circ}$ increments and 1 -s acquisitions at $30 \mathrm{keV}$. Three-dimensional datasets were reconstructed from the planar images with an ordered subset-estimation maximization (OSEM) algorithm (11).

SPECT images were obtained from a maximum likelihoodexpectation maximization (MLEM) reconstruction of 60 projections at $6^{\circ}$ intervals over $360^{\circ}$ of rotation and 30- or $60-\mathrm{s}$ acquisitions $(12,13)$.

\section{Image Display}

Amide imaging software was used to coregister the CT and SPECT datasets (14). CT and SPECT datasets were scaled by voxel size and manually aligned with simple rotation and translation operations using anatomic landmarks.

\section{RESULTS}

The size of the synthetic SPECT lesion was estimated optically with a dissecting microscope. Dimensions of the chamber housing the anion-exchange beads were $0.6 \mathrm{~mm}$ in diameter by 0.5 - to $1.5-\mathrm{mm}$ in length in multiple catheters containing 2-6 beads. The ion-exchange beads swell to a diameter of up to $750 \mu \mathrm{m}$ when wet and nearly fill the available 100-300 $\mathrm{nL}$ of space. Individual beads are at the limits of spatial resolution for our imaging systems and give an apparent lesion size approximately equal to the space in the bead chamber (Fig. 1).

The activity within the synthetic lesions depends on the number of beads, the activity applied to the column, and the decay fraction of ${ }^{99} \mathrm{TcO}_{4}{ }^{-}$. Typical values of $7.4-22.2$ $\mathrm{MBq}(0.2-0.6 \mathrm{mCi})$ were observed, with increasing activity correlating with more beads (Fig. 2). Planar scintigraphy demonstrated complete clearing of activity from the tubing and retention of the radiotracer only at the site of the beads (Fig. 2). After decay of the ${ }^{99 \mathrm{~m}} \mathrm{Tc}$ to ${ }^{99} \mathrm{Tc}$, the catheters were routinely reloaded with ${ }^{99} \mathrm{~m} \mathrm{TcO}_{4}{ }^{-}$by reinjecting the catheter and flushing with water.

The synthetic SPECT lesion was placed initially into an excised mouse femur to simulate a bone tumor. Images were acquired on the dual-modality system. The CT images demonstrate entrance of the catheter into the marrow space of the distal metaphysis and exit of the catheter from the distal diaphysis. SPECT images demonstrate localization of the ${ }^{99} \mathrm{mcO}_{4}{ }^{-}$activity to the site of the beads within the catheter (Fig. 3).

Subsequently, a similar catheter was placed in the femur of a live mouse. The mouse was imaged after administration of ${ }^{99 \mathrm{~m}} \mathrm{Tc}$-methylene diphosphonate (MDP) by tail-vein injection and charging of the synthetic lesion with ${ }^{99} \mathrm{TcO}_{4}{ }^{-}$. CT images demonstrate entrance and exit sites of the catheter in the lateral aspect of the distal femur. SPECT images demonstrate expected low-level radiotracer uptake in the mouse skeleton. Focally increased activity is seen at the right knee synthetic lesion (Fig. 4). The mouse tolerated the procedure and indwelling catheter extremely well, maintaining weight, normal activity, and grooming. No signs of infection developed while the mouse was imaged repeatedly on multiple imaging systems for over $8 \mathrm{wk}$.

The synthetic SPECT lesion was used in our laboratory to refine the coregistration of image sets obtained on the dual-modality system and to develop and refine a multiinstrument imaging scheme before a large-scale study of neuroblastoma metastasis to the femur.

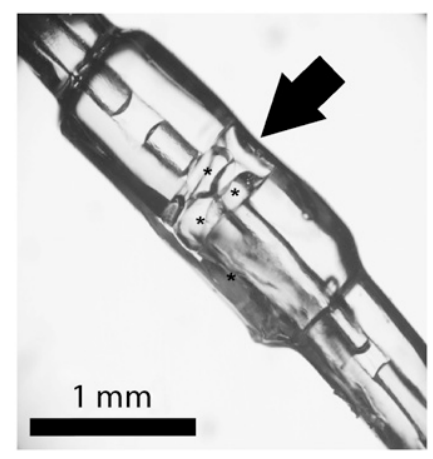

FIGURE 1. Photomicrograph of synthetic SPECT lesion catheter system containing 3 AG $1-X 2$ anionexchange chromatography beads (each bead marked by asterisk). Arrow points to chamber housing the beads. 


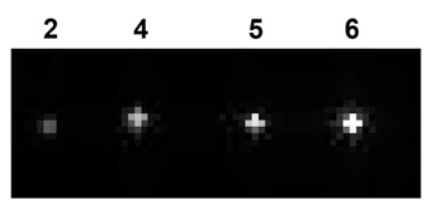

FIGURE 2. Planar scintigraphy of 4 catheters containing 2, 4, 5, and 6 beads from left to right.

\section{DISCUSSION}

Development and characterization of prototype imaging systems rely heavily on phantoms. Many phantoms are commonly used for system assessment, calibration, and maintenance - that is, Mini-Defrise and Mini-Jaszczak (Data Spectrum Corp.), Derenzo, etc. Most phantoms have little or no resemblance to the organ or animal to be imaged. Rigorous assessment of system performance for "real-life" tasks such as lesion detection requires task-based studies, and physical and digital phantoms have been developed to simulate anatomy, pathology, and dynamics and to evaluate system performance lesion detection $(15,16)$.

Novel small-animal imaging systems may be "fully" developed and characterized without ever imaging a live animal. Allowances for animal monitoring, support, anesthesia, and positioning may be incorporated late in the assembly stage. Design changes may become necessary when live animals are imaged. Despite the number and utility of phantoms available for assessment and optimization of imager performance, there have been few tools that bridge the gap between imaging phantoms and imaging live subjects. Although preliminary system optimization may be performed with healthy animals, an animal with a known lesion is desirable for optimization of the imager for lesion
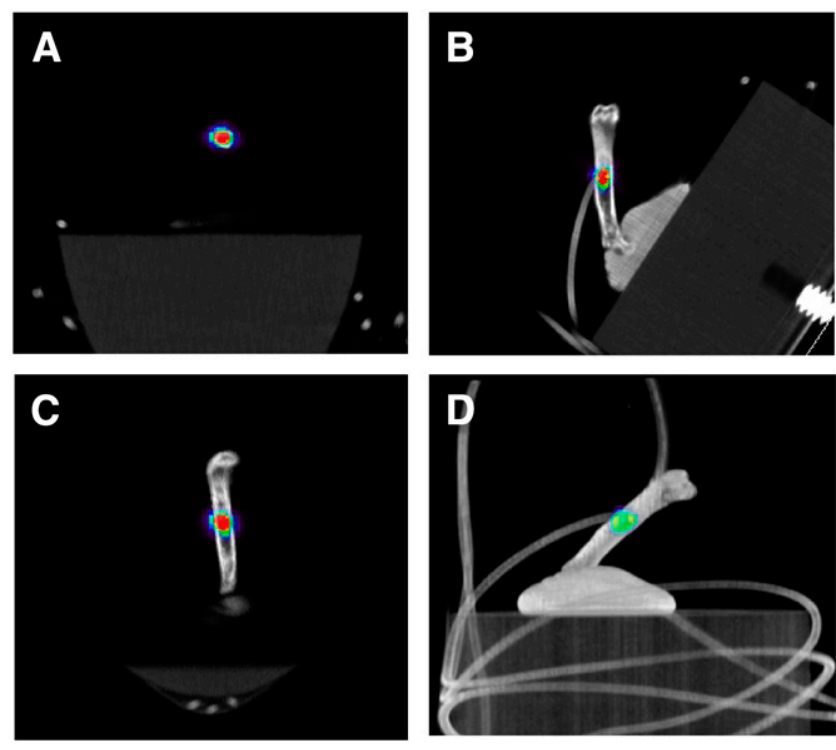

FIGURE 3. 99mTc SPECT/CT fusion images of synthetic SPECT lesion catheter implanted in excised mouse femur. Axial $(A)$, coronal $(B)$, and sagittal $(C)$ views through lesion demonstrate coregistration of CT and SPECT datasets. 3-dimensional surface rendering of entire femur (D) demonstrates entering and exiting catheters used to load, flush, and elute anion-exchange beads.

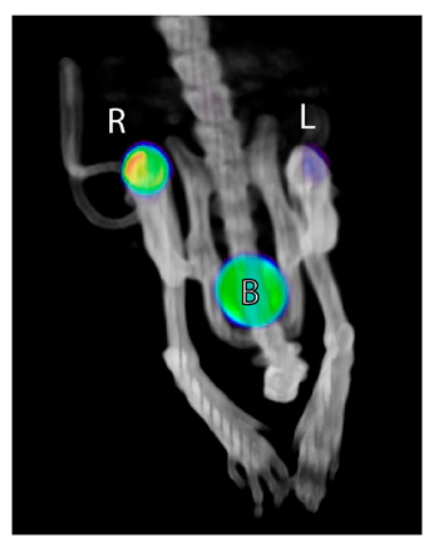

detection. The quantitative calibration of system sensitivity to permit determination of absolute uptake values can also be facilitated by a synthetic SPECT lesion.

There are several limitations to the implantable synthetic lesion. The catheter ends protrude from the skin and can be a source of irritation to the animal. An exit site at the nape of the neck was selected to minimize the mouse's ability to disturb the site. The synthetic lesion was constructed from chemically inert polyvinyl chloride tubing, which has acceptable biocompatibility in humans, to minimize the risk of foreign-body reactions in the mouse. An opening in the skin creates a potential route for infection, although we observed no signs of infection for up to $8 \mathrm{wk}$. Normal healing about the catheter entry site seems to provide an adequate barrier to common pathogens in the laboratory environment. Once the catheter has been placed, it cannot be repositioned without additional surgery. The catheter may clog or leak; flushing the catheter with water is essential to prevent precipitated salts from clogging the catheter, and a leaking catheter may be a risk to the animal because large volumes of fluid may be injected and result in volume overload.

The synthetic SPECT lesion was implanted in mouse femurs to simulate a bone tumor. The catheter may be implanted at other locations to simulate other lesions. The morbidity of implantation at other locations is not known and would require careful monitoring. The synthetic lesion is suitable for implantation in other animals. Larger animals, such as rats, could also accommodate a subcutaneous injection port for the catheter, allowing complete internalization of the device, further reducing the risk of infection and discomfort.

Although our efforts were focused on the development of small lesions to simulate early tumors and to challenge the sensitivity and resolution of our imaging systems, lesions of any size can be constructed. Larger lesions could be simulated by the selection of larger tubing sizes and incorporation of additional ion-exchange resin beads. Selection of a cation-exchange resin would allow construction of an ${ }^{111}$ In lesion to assess imaging system performance at higher photon energies. 


\section{CONCLUSION}

The synthetic implantable SPECT lesion is a biocompatible, realistically sized, reusable device that may be useful for many applications, including small-animal imaging system development and characterization.

\section{ACKNOWLEDGMENTS}

The authors thank Christy Barber for her expertise and skill in animal care and imaging. This work was supported by NIBIB grants P41EB002035 and P41EB002035-05S1.

\section{REFERENCES}

1. Weber DA, Ivanovic M. Pinhole SPECT: ultra-high resolution imaging for small animal studies. J Nucl Med. 1995;36:2287-2289.

2. Paulus MJ, Gleason SS, Kennel SJ, Hunsicker PR, Johnson DK. High resolution $\mathrm{x}$-ray computed tomography: an emerging tool for small animal cancer research. Neoplasia. 2000;2:62-70.

3. Herschman HR. Micro-PET imaging and small animal models of disease. Curr Opin Immunol. 2003;15:378-384.

4. Meikle SR, Kench P, Kassiou M, Banati RB. Small animal SPECT and its place in the matrix of molecular imaging technologies. Phys Med Biol. 2005;50:R45R61.
5. Beekman F, van der Have F. The pinhole: gateway to ultra-high-resolution threedimensional radionuclide imaging. Eur J Nucl Med Mol Imaging. 2007;34:151161.

6. Strand SE, Ivanovic M, Erlandsson K, et al. Small animal imaging with pinhole single-photon emission computed tomography. Cancer. 1994;73(3 suppl):981984.

7. Paulus MJ, Gleason SS, Easterly ME, Foltz CJ. A review of high-resolution x-ray computed tomography and other imaging modalities for small animal research. Lab Anim (NY). 2001;30:36-45.

8. Weber DA, Ivanovic M. Ultra-high-resolution imaging of small animals: implications for preclinical and research studies. J Nucl Cardiol. 1999;6:332-344.

9. Kastis GA, Wu MC, Balzer SJ, et al. Tomographic small-animal imaging using a high-resolution semiconductor camera. IEEE Trans Nucl Sci. 2002;49:172-175.

10. Kastis GA. Multi-Modality Imaging of Small Animals [dissertation]. Tucson, AZ: University of Arizona; 2002.

11. Hudson HM, Larkin R. Accelerated image reconstruction using ordered subsets of projection data. IEEE Trans Med Imaging. 1994;13:601-609.

12. Shepp LA, Vardi Y. Maximum likelihood estimation for emission tomography. IEEE Trans Med Imaging. 1982;1:113-121.

13. Lange $\mathrm{K}$, Carson R. EM reconstruction algorithm for emission and transmission tomography. J Comput Assist Tomogr. 1984;8:306-310.

14. Loening AM, Gambhir SS. AMIDE: a free software tool for multimodality medical image analysis. Mol Imaging. 2003;2:131-137.

15. Segars WP, Tsui BM, Frey EC, Johnson GA, Berr SS. Development of a 4-D digital mouse phantom for molecular imaging research. Mol Imaging Biol. 2004; 6:149-159.

16. Veress AI, Spears WP, Weiss JA, Tsui BM, Gullberg GT. Normal and pathological NCAT image and phantom data based on physiologically realistic left ventricle finite-element models. IEEE Trans Med Imaging. 2006;25:1604-1616. 